\title{
Sleep Disturbance Strongly Related to the Development of Postoperative Delirium in Proximal Femoral Fracture Patients Aged 60 or Older
}

\author{
Myung-Rae Cho, MD, Suk-Kyoon Song, MD, Cheol-Hwan Ryu, MD \\ Department of Orthopedic Surgery, Daegu Catholic University Medical Center, Daegu, Korea
}

Purpose: Post-fracture sleeping disorders can lead to a deterioration of mental and physical health and delay recovery to pre-fracture status. Here, an analysis was conducted to determine if sleep disturbance is a risk factor for delirium in patients older than 60 years of age with surgically treated proximal femoral fractures.

Materials and Methods: This retrospective study included 316 patients with surgically treated proximal femoral fractures between January 2014 and December 2016; 33 patients were removed from analysis due to exclusion criteria. Confirmation of delirium was made by a neurologist upon consultation for cognitive impairment and sleeping disorders were confirmed by a doctor or nurse based on the Pittsburgh sleep quality index. Potential risk factors other than a sleep disorder (e.g., history of cognitive impairment, medical illness, preoperational levels of albumin and hemoglobin, transfusion) were also analyzed as variables for the development of delirium.

Results: The sensitivity and specificity of a sleeping disorder as a risk factor for the development of delirium were 0.75 and 0.76 , respectively; the positive and negative predictive values were 0.64 and 0.93 , respectively. A sleeping disorder was significantly related to the development of the delirium (odds ratio adjusted for age, sex and body mass index was $5.78, P<0.01)$. In those with a history of cognitive impairment, the adjusted odds ratio for the development of delirium was $6.03(P<0.01)$.

Conclusion: Sleeping disorders occurring after a surgically repaired proximal femoral fracture in patients 60 years of age or older could be an independent predictive factor of delirium.

Key Words: Hip fractures, Sleep, Delirium

Submitted: December 30, 2019 1st revision: March 26, 2020

Final acceptance: April 7, 2020

Address reprint request to

\section{Suk-Kyoon Song, MD}

(https://orcid.org/0000-0002-3241-5820)

Department of Orthopedic Surgery, Daegu Catholic University Medical Center, 33 Duryugongwon-ro 17-gil, Nam-gu, Daegu

42472, Korea

TEL: +82-53-650-4277 FAX: +82-53-626-4272

E-mail: ryansong10anaver.com

This is an Open Access article distributed under the terms of the Creative Commons Attribution Non-Commercial License (http://creativecommons. org/licenses/by-nc/4.0) which permits unrestricted non-commercial use, distribution, and reproduction in any medium, provided the original work is properly cited.

\section{INTRODUCTION}

Delirium is one of the most common complications for elderly patients with proximal femoral fractures ${ }^{1-3)}$. The incidence rate of delirium after a proximal femoral fracture varies drastically by race (from $4-61 \%)^{2}$. Delirium, which is a neurobehavioral syndrome in which normal neural activity is altered, can be caused by a variety of mechanisms ${ }^{4}$. Delirium is associated with longer hospital stays, worse prognoses, and increases in healthcare expenses and mortality rates in senile patients with proximal femoral fractures ${ }^{1,5}$. Known risk factors of delirium are being 65 years of age or older, male sex and dementia or depression ${ }^{6}$, however other 


\section{Hip \& Pelvis}

Hip Pelvis 32(2): 93-98, 2020

risk factors may also exist (e.g., transfusion, type of fracture, type of operation, American Society of Anesthesiologists [ASA] classification, body mass index [BMI], time until operation after fracture, preoperative albumin levels, preoperative hemoglobin levels). It is also possible that a post-fracture sleep disorder may be the cause of delirium, with secondary symptoms developed as a result of the delirium $^{7}$, however, studies to date have been insiufficient to confirm this possibility. Here, the potential relationship between sleeping disorders and delirium in patients with surgically treated proximal femoral fractures was investigated. The hypothesis tested here was that a sleeping disorder occurring after surgical treatment of a proximal femoral fracture could be a risk factor for the development of delirium. Thus, the objective of this study was to characterize the relationship between sleep disorders and the development of delirium in patients with surgically treated proximal femoral fractures.

\section{MATERIALS AND METHODS}

\section{Study Design and Patient Selection}

This retrospective study included 316 patients with proximal femoral fractures treated with surgery between January 2014 and December 2016 (Fig. 1); 33 patients were removed from analysis based on exclusion criteria (i.e., under 60 years of age, a cognitive disorder at admission, admission in the intensive care unit, no surgery). Patients with a history of dementia or abnormal cognitive function were included if the results of a neurology consultation were normal. The proximal femoral fractures included here were a mix of intertrochanteric and femoral neck fractures and they were managed with total hip arthroplasty, bipolar hemiarthroplasty or internal fixation. Delirium diagnoses were made based on behavioral observation and cognitive assessment. The confusion assessment method (CAM) was used to screen for delirium in this study ${ }^{8}$. CAM consists of 4 core features of delirium (acute onset and fluctuating

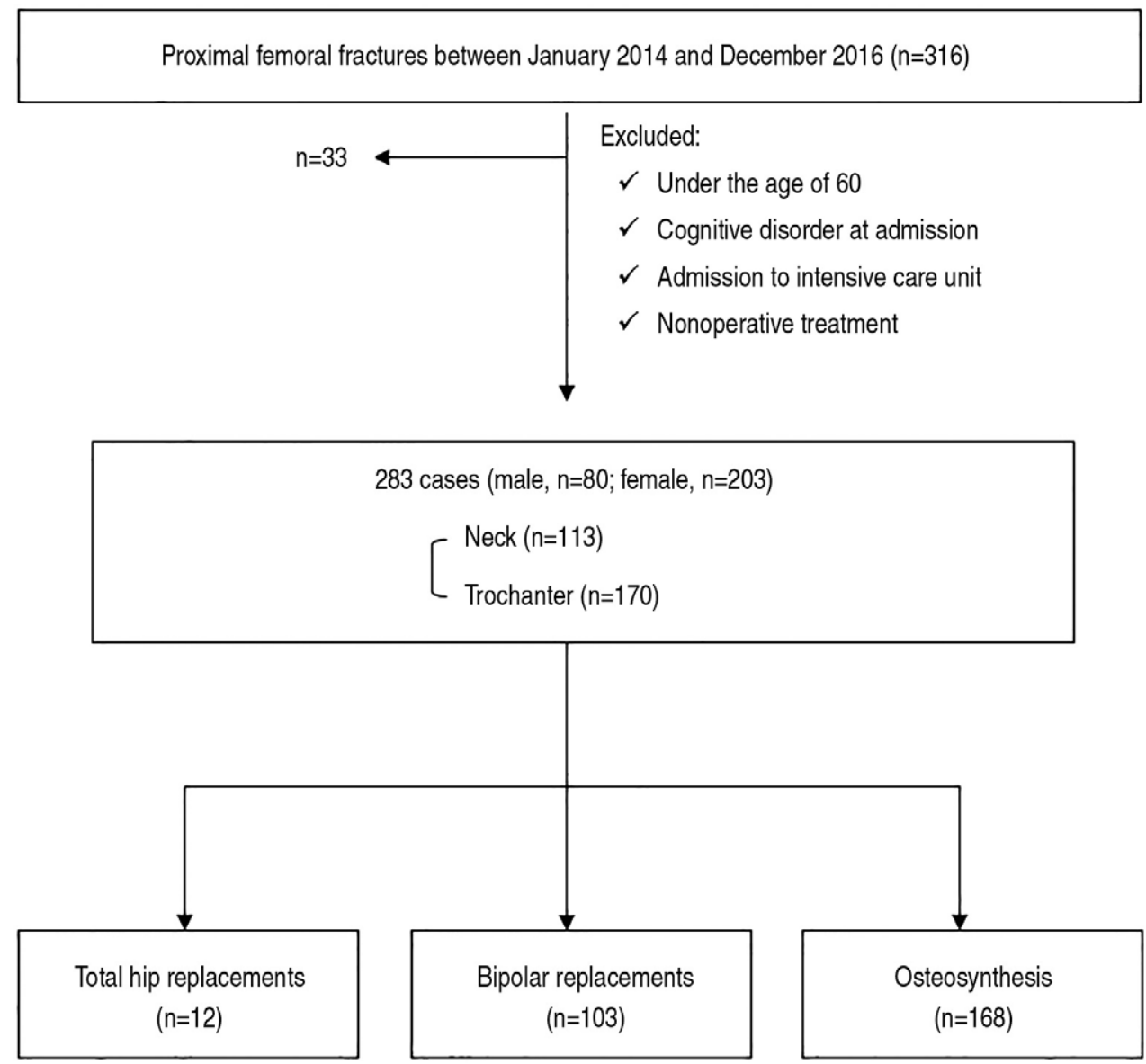

Fig. 1. Flow chart of patients with proximal male/female fracture. 
course of symptoms, inattention, and either disorganized thinking or altered level of consciousness) which are assessed by a trained neurologist to make a definite diagnosis (Table 1). Sleeping disorders can be assessed using the Pittsburgh sleep quality index (PSQI); many studies have used this tool to assess sleep quality in sleeping disorders ${ }^{9}$. The original PSQI-validation study indicated that a cut-off score of $\geq 5$ can be used to identify poor sleepers ${ }^{10)}$. Since a sleeping disorder may be a secondary symptom of delirium, patients with sleeping disorders diagnosed at the same time or after delirium were excluded. Other than sleeping disorders, the potential relationship between occurrence of delirium and other variables (i.e., history of cognitive impairment, transfusion, sex, type of fracture, type of operation, ASA classification, age, BMI, time until operation after fracture, preoperative albumin level, preoperative hemoglobin) was investigated. The study was approved by the Institutional Review Board (CR19-133).

\section{Statistical Analysis}

To analyze the potential relationship between postoperative occurrence of delirium and sleeping disorder, fracture management approach, history of dementia, history of cognitive impairment, transfusion, sex, fracture type, operation type, ASA classification, age, BMI, time until operation after fracture, preoperative albumin level, and preoperative hemoglobin, the Fisher test and Student ttest were used. A multivariable logistic regression test was also used to grade the relationship between risk factors and delirium. Statistical significance was set as a $P$-value of less than 0.05. Analyses were conducted using IBM SPSS Statistics ver. 19 (IBM Crop., Armonk, NY, USA).

\section{RESULTS}

Among the 283 patients included in the final analysis (i.e., after removal of 33 for exclusion criteria described above), 170 had intertrochanteric fractures and 113 had femoral neck fractures. Reconstructive surgery was used in 115 patients (total hip arthroplasty [n=12] and hemiarthroplasty $[n=103])$, and 168 underwent internal fixation for osteosynthesis. There were 28 patients $(9.9 \%)$ with a history of dementia (Table 2). After the fracture, more than one third of patients $(\mathrm{n}=101 ; 35.7 \%)$ were diagnosed with sleeping disorders and $48(17.0 \%)$ demonstrated delirium (Table 3 ). The sensitivity and specificity of a sleeping disorder to predict the development of delirium were 0.75 and 0.76 , respectively; the positive and negative predictive values were 0.64 and 0.93 , respectively. The mean time to operation after fracture was $5.77 \pm 3.58$ days; neurology consultation for the management of delirium occurred at a mean of $7.25 \pm 3.22$ days after fracture. Among the predicted risk factors for delirium, only sleeping disorder and history of dementia were significantly related to the development of delirium $(P<0.05)$. Odds ratios were 7.85 for sleeping disorder and 7.76 for history of dementia. The remaining predicted risk factors tested were not significantly related to the development of delirium. The adjusted odds ratio with sex, age, and BMI is 5.78 for those with a sleeping disorder and the development of dementia, a relationship that is statistically significant $(P<0.01)$. Those who had a history of dementia, the adjusted odds ratio

Table 1. Confusion Assessment Method (CAM)

\begin{tabular}{|c|c|}
\hline $\begin{array}{l}\text { 1: Acute onset and } \\
\text { fluctuating course }\end{array}$ & $\begin{array}{l}\text { - Is there evidence of an acute change in mental status from the patient's baseline? } \\
\text { - Did the labnormall behavior fluctuate during the day, that is, tend to come and go, or } \\
\text { increase and decrease in severity? }\end{array}$ \\
\hline 2: Inattention & $\begin{array}{l}\text { Did the patient have difficulty focusing attention, for example, being easily distractible, } \\
\text { or having difficulty keeping track of what was being said? }\end{array}$ \\
\hline 3: Disorganized thinking & $\begin{array}{l}\text { Was the patient's thinking disorganized or incoherent, such as rambling or irrelevant } \\
\text { conversation, unclear or illogical flow of ideas, or unpredictable switching from subject } \\
\text { to subject? }\end{array}$ \\
\hline $\begin{array}{l}\text { 4: Altered level of } \\
\text { consciousness }\end{array}$ & $\begin{array}{l}\text { Overall, how would you rate this patient's level of consciousness? } \\
\text { - Alert [normal], } \\
\text { - Vigilant [hyper-alert], } \\
\text { - Lethargic [drowsy, easily aroused], } \\
\text { - Stupor [difficult to arouse], or } \\
\text { - Coma [unarousable] }\end{array}$ \\
\hline
\end{tabular}

The diagnosis of delirium by CAM requires the presence of features 1 and 2 and either 3 or 4 . 


\section{Hip \& Pelvis}

Hip Pelvis 32(2): 93-98, 2020

with sex, age, and BMI for the development of delirium is 6.03, also statistically significant $(P<0.01)$ (Table 4).

\section{DISCUSSION}

The reported prevalence rates for delirium after a proximal femur fracture varies greatly depending on the study (4$61 \%)^{2)}$; this wide range can be explained, at least in part, by the definition of delirium used ${ }^{11}$. In this retrospective study, delirium was diagnosed by a neurologist after an assessment of potential cognitive impairment. The observed incidence rate of delirium in this study is $17 \%$, a value similar to a study by Inouye et al. ${ }^{12)}$, but one which could also be underestimated if a neurology consultation was not obtained. Kim and $\mathrm{Kim}^{13)}$ classified delirium into those resulting from either predisposing causes or trigger causes; predisposing causes include dementia or cognitive disorder, decreased performance ability, visual field defect, history of alcohol abuse and age older than 70 and trigger causes include dehydration, hypoxia, ischemia, infection and fracture, sleeping disorder, and environmental factors (e.g., intensiive care unit [ICU] care $)^{14}$. In addition, Altman et

Table 2. Patient's Demographs

\begin{tabular}{lc}
\hline \hline Variable & Result \\
\hline Age (yr) & $78.73 \pm 7.33$ \\
Sex (male/female) & $80(28.3) / 203(71.7)$ \\
Body mass index (kg/m) & $21.50 \pm 3.55$ \\
Time to operation (day) & $5.77 \pm 3.58$ \\
Initial albumin (g/dL) & $3.45 \pm 0.42$ \\
Initial hemoglobin (g/dL) & $11.08 \pm 1.32$ \\
Initial hematocrit (\%) & $33.20 \pm 4.01$ \\
Fracture classification (femur neck/intertrochanteric) & $113(39.9) / 170(60.1)$ \\
Past history of dementia (yes/no) & $28(9.9) / 255(90.1)$ \\
ASA classification (1/2/3) & $9(3.2) / 250(88.3) / 24(8.5)$ \\
\hline
\end{tabular}

Values are presented as mean \pm standard deviation or number $(\%)$.

ASA: American Society of Anesthesiologists.

Table 3. Relationship between Sleeping Disorder and Delirium

\begin{tabular}{lrrr}
\hline \hline & \multicolumn{3}{c}{ Sleeping disorder } \\
\cline { 2 - 3 } Variable & No & Yes & \\
\hline Delirium & & & 65 \\
No & 170 & 36 & 235 \\
Yes & 12 & 101 & 48 \\
Total & 182 & 283 \\
\hline
\end{tabular}

Values are presented as number only.

Table 4. Analysis of Multiple Variables Effects for Possible Risk Factors for Delirium

\begin{tabular}{lccccc}
\hline \hline Variable & $P$-value & $\begin{array}{c}\text { Crude odds } \\
\text { ratio }\end{array}$ & $\begin{array}{c}\text { Adjusted } \\
\text { odds ratio* }\end{array}$ & $\begin{array}{c}\text { 95\% confidence } \\
\text { lowest }\end{array}$ & $\begin{array}{c}\text { 95\% confidence } \\
\text { highest }\end{array}$ \\
\hline Sleeping disorder & $<0.01$ & 7.85 & 5.78 & 2.66 & 12.54 \\
History of dementia & $<0.01$ & 7.76 & 6.03 & & 15.48 \\
Age & 0.155 & 1.032 & & \\
Body mass index & 0.383 & 1.039 & & \\
Time until operation & 0.924 & 0.996 & & \\
Albumin & 0.966 & 0.984 & & \\
Type of fracture & 0.484 & 0.794 & & \\
Transfusion & 0.258 & 0.682 & & & \\
\hline
\end{tabular}

*Adjusted odds ratio with sex, age, and body mass index. 
al. ${ }^{7)}$ reported that higher ASA scores prior to operation and preoperational delirium are potential causes of postoperational delirium. Fong et al. ${ }^{4)}$ stated that impaired hearing and vision, insertion of catheter, activity restriction with restraints, medication, acute neuropathy (e.g., cerebral infarction, encephalitis), infection, anemia, disease, such as dehydration, operation, ICU care, sleeping disorder and pain are modifiable factors. In contrast, there are factors that could not be modified such as dementia, old age, history of delirium before, cerebral infarction, male, chronic neuropathy, and chronic liver disease. As seen from previous studies, a sleeping disorder is a predisposing factor or risk factor that can be modified to prevent delirium. However, to date, there has been insufficient research on the concomitant incidence of delirium and a sleeping disorder. Here, it was observed that if a patient has history of dementia, they are 6.03 times more likely to have delirium after a proximal femoral fracture. Patients with sleeping disorders after proximal femoral fracture have an adjusted odds ratio of 5.78 of experiencing delirium compared with those who does not.

This study has several limitations. First, it is a retrospective study using electronic medical records charts, thus the dates of delirium and sleeping disorder occurrence may be inaccurate due to negligence of recording. Second, since this study covers the elderly, sub-acute delirium may be misdiagnosed as a deconditioning postoperative event. Third, delirium could be underestimated when symptoms involved hypoactive conditions. However, rounds were made every day, and efforts were made to check patient conditions daily. Fourth, there were several inefficiencies when defining a sleeping disorder- polysomnography, electrocephalogram, and blood testing were not used to identify the cause. A self-reported questionnaire (PSQI) was used to assess sleep quality. Fifth, sleeping problems can be due to a chronic illness described on Charlson's comorbidity index, such as diabetes, arthritis, human immunodeficiency virus/acquired immunodeficiency syndrome, lupus, Parkinson's disease, Alzheimer's disease, and multiple sclerosis. There were a few comparable cases with the Charlson's comorbidity score. This system has greater advantages for the prediction of mortality, but relatively less well characterized for older patients because lots of index diseases are unnecessary. The ASA score system was also used for chronic illness as variants. Further prospective studies may be needed to overcome such limitations.

\section{CONCLUSION}

Our study suggests that in proximal femur fracture patients aged 60 or older, sleeping disorders which occur after surgery are significantly related to and an independent predictive factor for the development of delirium.

\section{CONFLICT OF INTEREST}

The authors declare that there is no potential conflict of interest relevant to this article.

\section{REFERENCES}

1. de Jong L, van Rijckevorsel VAJIM, Raats JW, Klem TMAL, Kuijper TM, Roukema GR. Delirium after hip hemiarthroplasty for proximal femoral fractures in elderly patients: risk factors and clinical outcomes. Clin Interv Aging. 2019;14:427-35.

2. Robertson BD, Robertson TJ. Postoperative delirium after hip fracture. J Bone Joint Surg Am. 2006;88:2060-8.

3. Kim KH, Kho DH, Shin JY, Choi JY, Kim ES, Kim DH. Risk factors of postoperative delirium in elderly patients with hip fractures. J Korean Fract Soc. 2008;21:189-94.

4. Fong TG, Tulebaev SR, Inouye SK. Delirium in elderly adults: diagnosis, prevention and treatment. Nat Rev Neurol. 2009;5:210-20.

5. Arshi A, Lai WC, Chen JB, Bukata SV, Stavrakis AI, Zeegen EN. Predictors and sequelae of postoperative delirium in geriatric hip fracture patients. Geriatr Orthop Surg Rehabil. 2018;9:2151459318814823.

6. Francis J. Delirium in older patients. J Am Geriatr Soc. 1992;40:829-38.

7. Altman MT, Knauert MP, Murphy TE, Ahasic AM, Chauhan Z, Pisani MA. Association of intensive care unit delirium with sleep disturbance and functional disability after critical illness: an observational cohort study. Ann Intensive Care. 2018;8:63.

8. Inouye SK, van Dyck CH, Alessi CA, Balkin S, Siegal AP, Horwitz RI. Clarifying confusion: the confusion assessment method. A new method for detection of delirium. Ann Intern Med. 1990;113:941-8.

9. Mollayeva T, Thurairajah P, Burton K, Mollayeva S, Shapiro CM, Colantonio A. The Pittsburgh sleep quality index as a screening tool for sleep dysfunction in clinical and non-clinical samples: a systematic review and metaanalysis. Sleep Med Rev. 2016;25:52-73.

10. Buysse DJ, Reynolds CF 3rd, Monk TH, Berman SR, Kupfer DJ. The Pittsburgh Sleep Quality Index: a new instrument for psychiatric practice and research. Psychiatry Res. 1989; 28:193-213.

11. Bruce AJ, Ritchie CW, Blizard R, Lai R, Raven P. The incidence of delirium associated with orthopedic surgery: a meta-analytic review. Int Psychogeriatr. 2007;19:197214.

12. Inouye SK, Westendorp RG, Saczynski JS. Delirium in 


\section{Hip \& Pelvis}

Hip Pelvis 32(2): 93-98, 2020

elderly people. Lancet. 2014;383:911-22.

13. Kim NY, Kim YW. Diagnosis and treatment of post-stroke delirium. Brain Neurorehabil. 2015;8:59-64.
14. Mistraletti G, Carloni E, Cigada M, et al. Sleep and delirium in the intensive care unit. Minerva Anestesiol. 2008;74: 329-34. 